\title{
Síntesis de zeolita LTA sobre soportes de corindón: Evaluación preliminar para la eliminación de metales pesados de efluentes acuosos
}

\author{
A. JACAS ${ }^{1 *}$, P. ORTEGA ${ }^{2}$, M.J. VELASCO², M.A.CAMBLOR', M.A. RODRÍGUEZ ${ }^{2}$ \\ ${ }^{1}$ Instituto de Ciencias de Materiales de Madrid. CSIC, Cantoblanco 28049, Madrid, España. \\ ${ }^{2}$ Instituto de Cerámica y Vidrio. CSIC, Cantoblanco 28049, Madrid, España
}

\begin{abstract}
En este trabajo se evalúa de forma preliminar la efectividad del empleo de materiales basados en zeolita LTA, como fase activa para su incorporación en sistemas orientados a la eliminación de metales pesados presentes en aguas residuales. Este tipo de zeolitas con el canal principal de un diámetro mínimo libre de $0,41 \mathrm{~nm}$ y una baja relación $\mathrm{SiO}_{2} / \mathrm{Al}_{2} \mathrm{O}_{3}$ se presenta como un tamiz molecular interesante, que a su vez manifiesta una gran capacidad de intercambio iónico. Partiendo de este punto de vista, cristales de zeolita LTA fueron obtenidos "in situ" por síntesis hidrotermal y caracterizados por difracción de rayos X (DRX), y microscopia electrónica de barrido (MEB). Se ha estudiado el efecto del tiempo de tratamiento hidrotermal a $378 \mathrm{~K}$. Asimismo, la capacidad de eliminación de metales pesados de la fase activa de los sistemas obtenidos fue evaluada en una primera etapa en soluciones diluidas de sales de cobre a $\mathrm{pH}$ ácido $(\sim 4,7)$.
\end{abstract}

Palabras claves: zeolita, membrana, síntesis hidrotermal, remoción, metales pesados, intercambio iónico.

\section{Synthesis of LTA zeolite on corundum supports: Preliminary assessment for heavy metal removal from waste water}

The effectiveness of materials based on LTA Zeolite as active phase, for their incorporation into systems aimed at the removal of heavy metals on waste water is evaluated in a preliminary way. This type of Zeolite with the main channel of a minimum free diameter of $0,41 \mathrm{~nm}$ and a low $\mathrm{SiO}_{2} / \mathrm{Al}_{2} \mathrm{O}_{3}$ ratio is an interesting molecular sieve, which in turn display a high ion exchange capacity. From this point of view, LTA Zeolite crystals were obtained "in situ" by hydrothermal synthesis and characterized by $x$ ray diffraction (XRD) and scanning electron microscopy (SEM). We have studied the effect of hydrothermal synthesis time at $378 \mathrm{~K}$. Likewise, the removal capacity of heavy metal from the active phase was evaluated in as a first step on diluted solutions of cooper salts at slightly acidic $\mathrm{pH}(\sim 4,7)$.

Key words: zeolite, membrane, hydrothermal synthesis, removal, heavy metals, ionic exchange.

\section{INTRODUCCIÓN}

Desde sus orígenes, la actividad de la raza humana ha sido la mayor responsable de la contaminación de las fuentes hídricas. Actualmente, esta situación plantea un problema global debido al incremento intensivo de la actividad tecnológica y de la vida cotidiana. $(1,2,3)$

En las últimas dos décadas, muchos estudios han estado dirigidos a poner de relieve la capacidad de diferentes sistemas y procesos en la eliminación de metales pesados de las aguas residuales y lodos. [4 -7]. Algunos de estos sistemas son muy caros y no son accesibles a los países en vías de desarrollo, aunque ciertos autores $(4,5,7,8)$ plantean la viabilidad técnica de diferentes adsorbentes de bajo coste para esos fines, en lugar del carbón activado, las resinas de intercambio iónico, etc., por citar algunos. En el curso de este período, muchos investigadores han estudiado diversos aspectos de la eliminación de metales pesados mediante el empleo de zeolitas y materiales en base a zeolitas. $(9-12)$

Las posibilidades de diseño "a medida" de zeolitas sintéticas de elevada pureza, en comparación a las variaciones que suelen manifestar las zeolitas naturales, contribuye a que las zeolitas sintéticas se empleen cada vez más, a pesar de ser más costosa su obtención.

Las zeolitas poseen estructura tridimensional, distribución de poros bien definida, alta superficie específica, y elevada resistencia química y térmica $(13,14)$. Su estructura presenta canales y/o cavidades de dimensiones moleculares y geometrías características para cada tipo de zeolita.

Debido a la sustitución isomórfica en la red de cationes $\mathrm{Si}^{4+}$ por $\mathrm{Al}^{3+}$, estas estructuras poseen una carga estructural negativa, que debe ser equilibrada por cationes intercambiables mono y divalentes en sitios activos específicos dentro de los canales, donde en función de su accesibilidad, pueden ser reemplazados por otros cationes, incluyendo metales pesados. $(4,15,16)$ La cantidad de cationes necesarios para mantener la electroneutralidad de la estructura será mayor cuanto mayor sea el grado de sustitución isomórfica en la red cristalina y por ende, mayor la capacidad de intercambio iónico. Desde esta perspectiva, las estructuras con una baja relación Si/Al 
y mayor accesibilidad al volumen interno son potencialmente las de mayor capacidad de intercambio iónico.

Las zeolitas sintéticas de baja relación $\mathrm{SiO}_{2} / \mathrm{Al}_{2} \mathrm{O}_{3}$ presentan usualmente tras la síntesis, cationes tales como $\mathrm{Na}^{+}, \mathrm{K}^{+}$. Estos cationes son necesarios para equilibrar la carga de la red aniónica originada por la coordinación tetraédrica de $\left[\mathrm{SiO}_{4 / 2}\right]^{+}$ y $\left[\mathrm{AlO}_{4 / 2}\right]$ conectados por medio de átomos de oxígeno.

Cuando los compuestos zeolíticos en forma sódica se introducen en soluciones acuosas que contienen otros cationes, se posibilita el intercambio de estos cationes por aquellos, siempre que puedan penetrar en los poros y cavidades, en función de sus dimensiones (incluyendo las moléculas de agua de coordinación de los cationes que se trate). El intercambio iónico que se realiza por tratamientos en fase líquida es considerado uno de los procesos principales para la eliminación de metales pesados $(17,18)$.

La eliminación por las zeolitas de los iones de metales pesados de las aguas residuales y lodos es un proceso controlado por el fenómeno de intercambio iónico, que puede ir acompañado también por el efecto de tamiz molecular de estos materiales. $(5,18-22)$

La aplicación potencial de las zeolitas para la eliminación de metales pesados varía en función del tipo de zeolita. La zeolita LTA con el diámetro libre mínimo en su canal principal de aproximadamente $0,41 \mathrm{~nm}$ de diámetro y una baja relación $\mathrm{SiO}_{2} / \mathrm{Al}_{2} \mathrm{O}_{3}$ hace de este material un tamiz molecular interesante, que manifiesta a su vez una gran capacidad de intercambio iónico y, por tanto, posee un alto potencial de aplicación en procesos de depuración de fases acuosas.

Algunos grupos han investigado la eliminación de $\mathrm{Cr}^{3+}$, $\mathrm{Cu}^{2+}, \mathrm{CO}^{2+}$, y otros cationes por intercambio iónico, empleando diferentes tipos de agregados zeolíticos, así como los efectos sobre esta capacidad en función de diferentes parámetros (24).

El objetivo de nuestro estudio se centra en la preparación y caracterización de la zeolita LTA como material activo. Considerando que las zeolitas de bajo contenido en sílice presentan la mayor capacidad de intercambio iónico, se ha sintetizado la zeolita LTA en las proximidades de la superficie porosa de soportes de alúmina por inmersión de los mismos en el gel y luego estos han sido sometidos a un proceso hidrotermal en diferentes intervalos de tiempo a presión autógena. Los precipitados testigos así obtenidos fueron caracterizados por difracción de rayos X (DRX) y microscopía electrónica de barrido (MEB).

\section{EXPERIMENTAL}

\subsection{Materiales y métodos}

Dos materiales distintos se han integrado en un sistema: por una parte, soportes de alúmina con una estrecha distribución de poros, buen comportamiento mecánico y estabilidad química y, por otra, cristales de zeolita embebidos "in situ", por síntesis hidrotermal en las proximidades de la superficie porosa del soporte de alúmina.

\subsubsection{PREPARACIÓN DE LOS SOPORTES DE ALÚMINA.}

Se emplean como soportes discos de $\alpha-\mathrm{Al}_{2} \mathrm{O}_{3}(35 \mathrm{~mm}$ diámetro, $4 \mathrm{~mm}$ de espesor, $1 \mu \mathrm{m}$ radio de poro, y $32 \%$ de porosidad). Estos soportes han sido obtenidos por prensado uniaxial de polvos de alúmina DK-206 (Martinswerk, Alemania, 95\%; $6 \mu \mathrm{m}$ tamaño medio de partícula; 1,3 $\pm 0,1$ $\mathrm{m}^{2} / \mathrm{g}$ de superficie específica) sometidos a cocción con una velocidad de calentamiento $5 \mathrm{~K} / \mathrm{min}$, hasta $1473 \mathrm{~K}$ durante 2 horas.

\subsubsection{SÍNTESIS DE LOS CRISTALES DE ZEOLITA LTA}

La síntesis hidrotermal a presión autógena se llevó a cabo por inmersión en posición vertical de los soportes de alúmina en el gel precursor de la zeolita, sin envejecimiento o siembra. En esta etapa el sistema fue sometido a tratamiento hidrotermal, a una temperatura de $378 \mathrm{~K}$ a diferentes intervalos de tiempo en reactores herméticos, confeccionados al efecto.

Los reactivos precursores empleados fueron pellets de hidróxido de sodio ( $\mathrm{NaOH}$, SIGMA-Aldrich, grado P.A.), silicato de sodio $\left(\mathrm{Na}_{2} \mathrm{O} \mathrm{SiO}_{2} .5 \mathrm{H}_{2} \mathrm{O}\right.$, SIGMA-Aldrich, grado P.A.), aluminato de sodio $\left(\mathrm{Na}_{2} \mathrm{OAl}_{2} \mathrm{O}_{3} \cdot 3 \mathrm{H}_{2} \mathrm{O}\right.$, SIGMA-Aldrich, grado P.A.) y agua desionizada.

La composición del sol fue tomada de un trabajo previo de Mark E. Davis y col. [25]

$$
34 \mathrm{Na}_{2} \mathrm{O}: \mathrm{Al}_{2} \mathrm{O}_{3}: 3 \mathrm{SiO}_{2}: 462 \mathrm{H}_{2} \mathrm{O}
$$

Para lograr la mezcla de reacción se preparan dos soluciones. La solución A se obtuvo por disolución de 4,36 g de $\mathrm{Na}_{2} \mathrm{OAl}_{2} \mathrm{O}_{3} \cdot 3 \mathrm{H}_{2} \mathrm{O}$ en $80 \mathrm{~g}$ de agua desionizada a una temperatura de $328 \mathrm{~K}$ hasta solubilidad total aparente y posterior adición de $24 \mathrm{~g}$ de $\mathrm{NaOH}$ mediante intensa agitación. Un segundo sol, denominado $\mathrm{B}$, se obtuvo por solución de $12,76 \mathrm{~g}$ de $\mathrm{Na}_{2} \mathrm{OSiO}_{2} \cdot 5 \mathrm{H}_{2} \mathrm{O}$ mediante agitación en $80 \mathrm{~g}$ de agua desionizada a $328 \mathrm{~K}$ y adición final hasta solubilidad aparente de $24 \mathrm{~g}$ de $\mathrm{NaOH}$.

En el procedimiento de Davis y col. [25] las soluciones se mantenían durante 24 horas a una temperatura de $268 \mathrm{~K}$ antes de iniciar la mezcla de reacción. En nuestro caso, una vez el sol B estuvo listo, fue vertido lentamente y mezclado mediante agitación con el A, hasta obtener una solubilidad aparente y luego trasvasado al reactor. A partir de este momento, pueden plantearse dos vías de actuación:

1. Tratamiento hidrotermal de la solución precursora en condiciones estáticas, a temperaturas de 373 ó $378 \mathrm{~K}$, a diferentes intervalos de tiempo (30, 60, 90 y $120 \mathrm{~min})$ bajo presión autógena.

2. Tratamiento hidrotermal de la solución precursora en condiciones estáticas con el soporte de alúmina "in situ" en posición vertical, a temperaturas de 373 ó 378 K, en los mismos intervalos de tiempo mencionados.

Concluido el tratamiento hidrotermal, el autoclave fue enfriado en una corriente de agua a temperatura ambiente. Se recuperaron los sólidos y los soportes de alúmina. Los sólidos precipitados fueron separados por filtración y lavados en múltiples ocasiones con agua desionizada hasta obtener un $\mathrm{pH}$ constante de las aguas de lavado. Los soportes de alúmina fueron sumergidos en abundante agua desionizada durante aproximadamente 240 minutos. Tanto los soportes de alúmina como los polvos recuperados se secaron a $303 \mathrm{~K}$ durante 12 horas. 


\subsubsection{ENSAYO DE ELIMINACIÓN DE METALES PESADOS.}

Se utilizó como material adsorbente el polvo de zeolita LTA, obtenido por tratamiento hidrotermal de la solución precursora a $378 \mathrm{~K}$ en el intervalo de tiempo correspondiente a una mayor manifestación de la estructura zeolítica LTA.

Como solución de ensayo se empleó una disolución acuosa de nitrato de cobre $0,02 \mathrm{~N}$ obtenida a partir de $\mathrm{Cu}\left(\mathrm{NO}_{3}\right)_{2} \cdot 3 \mathrm{H}_{2} \mathrm{O}$ (MERCK, grado P.A.).

El ensayo de eliminación de metales pesados por intercambio iónico fue llevado a cabo por agitación continua a $180 \mathrm{rev} / \mathrm{min}$ de una serie de frascos herméticamente cerrados que contenían $100 \mathrm{ml}$ de la disolución acuosa antes mencionada, a pH de aproximadamente 4,7, a los que se adicionó $1 \mathrm{~g}$ del producto zeolítico, para una relación de disolución : sólido intercambiador 100:1. El estudio fue realizado a una temperatura de $298 \pm 2 K$, para intervalos de tiempo de 0,$5 ; 1,2$, 4, 8 y 24 horas. Transcurridos estos intervalos, las muestras fueron filtradas al vacío: las aguas de filtración almacenadas a $277 \mathrm{~K}$ para su posterior análisis químico y los sólidos se secaron en estufa a $323 \mathrm{~K}$ para su posterior análisis por DRX.

La agitación continua fue utilizada con el objetivo de garantizar que la fase activa, atendiendo a su densidad, no se depositara paulatinamente en el fondo del recipiente. Esta agitación continua puede favorecer el proceso de difusión de los iones de $\mathrm{Cu}^{2+}$ dentro de los microporos.

La eficiencia de la eliminación fue evaluada en cada intervalo de tiempo según la relación (10):

$$
R(\%)=\left[\frac{C_{o}-C_{e q}}{C_{o}}\right] .100
$$

Siendo:

C: concentración inicial

$C_{e q}$ : supuesta concentración de equilibrio.

\section{CARACTERIZACIÓN}

La caracterización de la composición de fases presentes en el producto zeolítico se llevó a cabo por difracción de rayos $\mathrm{x}$ (DRX), método de polvos, a partir del estudio de los patrones de difracción obtenidos en un difractómetro Bruker D8 ADVANCE, empleando radiación $\mathrm{CuK}_{\alpha}$ y barrido en el intervalo $4^{\circ}<2 \vartheta<50^{\circ}$ con un paso de $0,05(2 \vartheta)$ a razón de 1,5 s/ paso.

Los soportes de alúmina tratados hidrotermalmente fueron fracturados y las microestructuras se analizaron con el empleo de un microscopio electrónico de barrido HITACHI S-4700. Este estudio suministró evidencias de los aspectos morfológicos de nuestro sistema.

En el análisis de la concentración de $\mathrm{Cu}(\mathrm{mg} / \mathrm{l})$ de la disolución original y de las disoluciones obtenidas después de los tratamientos con la zeolita, se ha utilizado la técnica de Espectroscopía de emisión atómica mediante Plasma de Acoplamiento Inductivo (ICP-EOS), empleando una curva de calibrado con tres patrones. Para ello se recurrió a un espectrómetro Thermo Jarrell Ash, modelo Iris Advantage, con sistema de visualización axial y óptica auxiliar con fuente de radiofrecuencia de 40,68 $\mathrm{MHz}$, red de difracción tipo Echelle, dispersión cruzada por prisma y un mecanismo de inyección de carga (CID) como detector de estado sólido.

\section{RESULTADOS Y DISCUSIÓN}

\subsection{Síntesis hidrotermal. Efecto del tiempo de reacción}

El polvo recuperado del producto de la síntesis realizada a $30 \mathrm{~min}$. de tratamiento hidrotermal es bajo, representando el $10 \%$ respecto del recuperado a los $120 \mathrm{~min}$. Con el incremento del tiempo de tratamiento hidrotermal aumenta la cantidad de fase sólida obtenida y, por consiguiente, el rendimiento.

Los procesos de nucleación y crecimiento son típicamente inducidos por fuerzas motrices vinculadas a la sobresaturación y al tiempo de reacción durante el tratamiento hidrotermal a presión autógena. Un aumento del tiempo de reacción debe manifestar el tránsito hacia fases más estables. La figura 1 muestra la influencia del tiempo de reacción sobre la composición de fases de los polvos obtenidos durante el proceso de síntesis hidrotermal. Los diferentes difractogramas fueron comparados entre si y con respecto a patrones de difracción de polvos simulados según Higgins y Treacy (28).

Los difractogramas muestran cambios en la intensidad de las reflexiones, como consecuencia de la evolución de los procesos de tránsito de fase. Los polvos obtenidos a intervalos de 60 y $75 \mathrm{~min}$. no muestran diferencias significativas entre las posiciones de las reflexiones, aunque sí en su intensidad. No obstante, en ambos la fase mayoritaria presente se corresponde con la estructura zeolítica tipo LTA.

Para intervalos superiores a 75 min se advierte un tránsito desde la estructura zeolítica tipo LTA hacia una fase más estable, como es la sodalita (SOD), que posee una menor capacidad de intercambio iónico debido a su menor tamaño de poro. El difractograma correspondiente al intervalo de 120 min muestra la coexistencia de las fases LTA y SOD con reflexiones importantes en $2 \theta=14,35$ y 43,08 grados. En todos los casos se observa una adecuada cristalinidad y, por tanto, es despreciable la contribución de la fracción amorfa de la muestra.

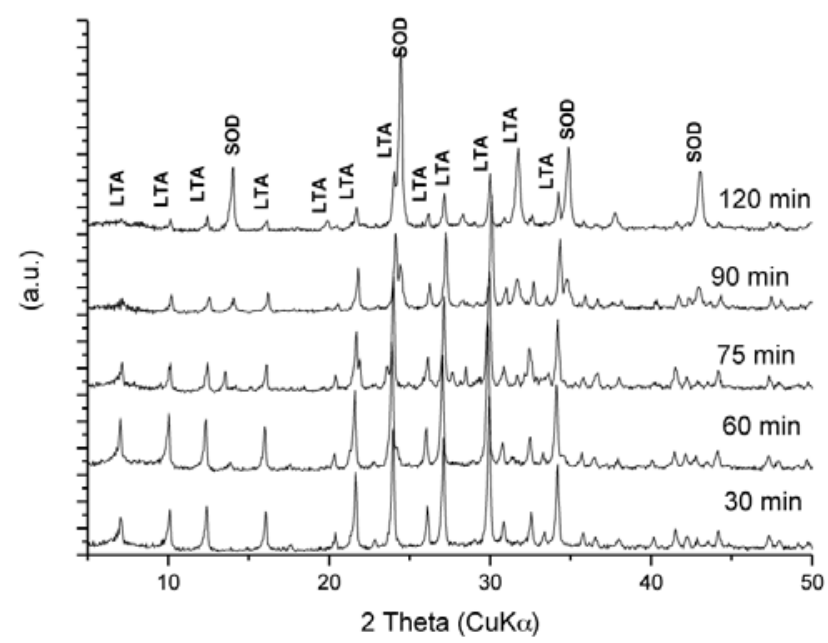

Fig. 1 Espectros de DRX de los polvos testigo sintetizados a diferentes intervalos de tiempo: 30, 60, 75, 90 y 120 minutos. Se pueden apreciar reflexiones características correspondientes a las fases LTA y sodalita (SOD) 


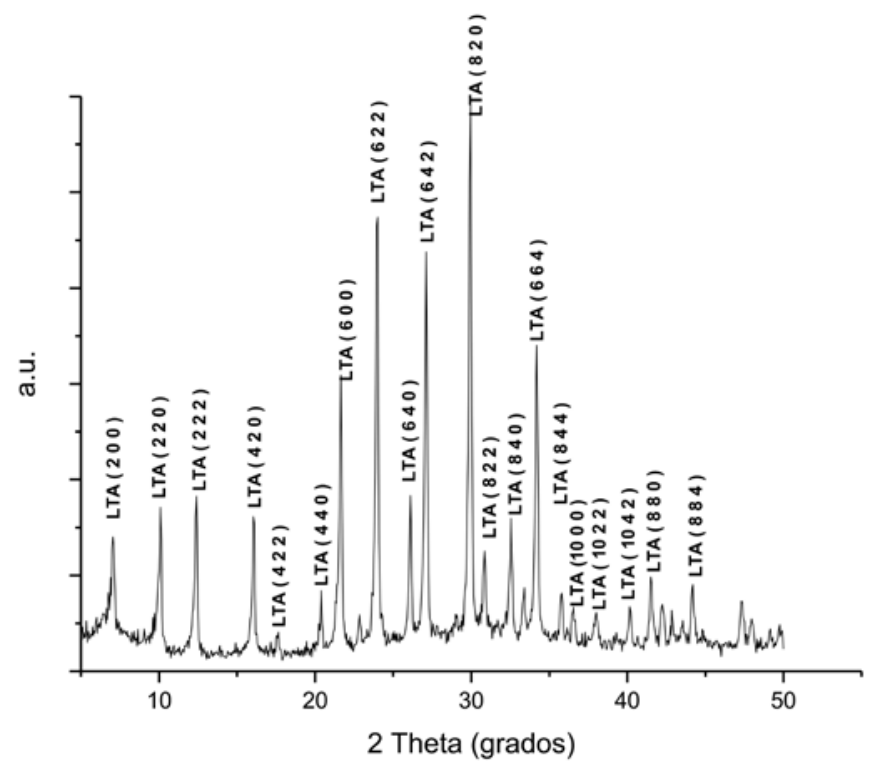

Fig. 2. Espectros de DRX del aluminosilicato sintetizado a partir de $34 \mathrm{Na}_{2} \mathrm{O}: \mathrm{Al}_{2} \mathrm{O}_{3}: 3 \mathrm{SiO}_{2}: 462 \mathrm{H}_{2} \mathrm{O}$, a $378 \mathrm{~K}$ en el intervalo de $75 \mathrm{~min}$.

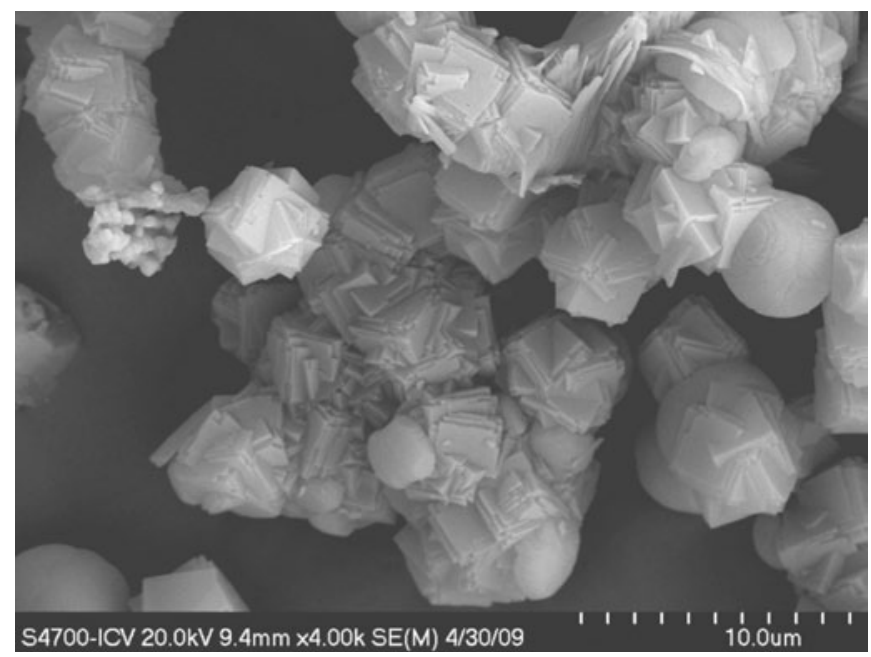

Fig.3. Imagen por MEB de los polvos de zeolita obtenidos por síntesis hidrotermal en el intervalo de $75 \mathrm{~min}$. Se destaca la formación de maclas con predominio de la forma cúbica característica de la Na-LTA.

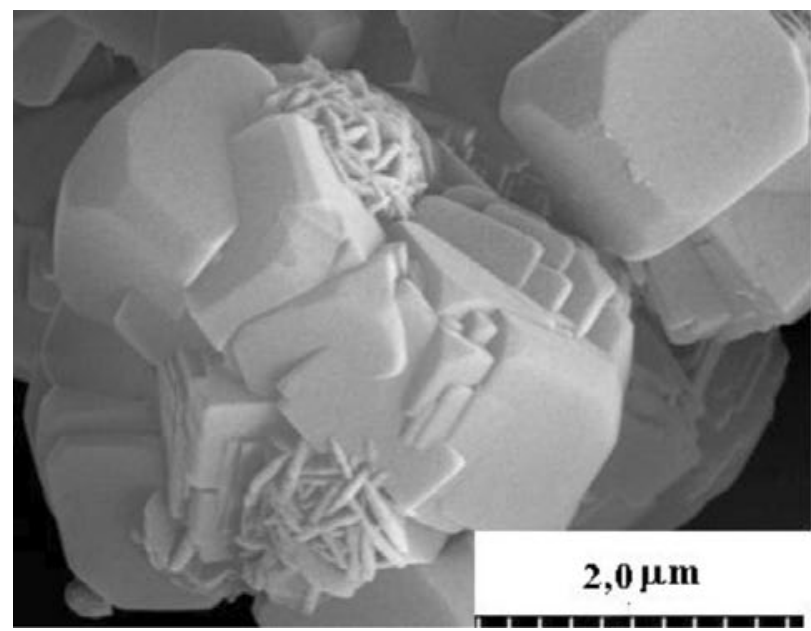

En el intervalo de tratamiento de $30 \mathrm{~min}$, la muestra presenta una estructura típica de la LTA, pero su rendimiento es el $10 \%$, comparado con el obtenido en el intervalo de 120 $\min$.

Considerando el rendimiento de la síntesis y la presencia de las diferentes fases zeolíticas, el tiempo adecuado para el desarrollo de la etapa de síntesis hidrotermal es el intervalo de 75 min. La asignación de los correspondientes índices $h k l$ a cada reflexión identificada (ver la Fig.2), permite corroborar que el material zeolítico obtenido en ese intervalo se corresponde mayoritariamente con una Na-LTA.

La figura 3 muestra la imagen obtenida por MEB de polvos sintetizados por tratamiento hidrotermal en el intervalo de 75 min. Los cristales se presentan formando maclas, en las cuales es destacable la apariencia cúbica en correspondencia con la simetría de la Na-LTA, que cristaliza teóricamente en el grupo espacial Fm-3c

También se observan aglomerados de forma esférica sin aparentes planos de reflexión que, en principio, pueden corresponder a reservorios de material, a partir de los cuales se nutre la formación de las estructuras características de la Na-LTA durante el proceso de cristalización posterior a la nucleación inicial y a la precipitación.

\subsection{Formación de Na-LTA en la superficie de los poros de los soportes cerámicos.}

En la figura 4 se observan las imágenes obtenidas por MEB de secciones de los soportes de alúmina tratados hidrotermalmente en la solución precursora. Los cristales de alúmina mostrados en la Fig.4a tienen el aspecto de placas superpuestas, manifestación característica de este tipo de material. Estos cristales de alúmina están recubiertos por pequeños cristales de zeolita de dimensiones que oscilan entre 0.5 y $1 \mu \mathrm{m}$, mostrando un aspecto morfológico propio de cubos biselados (14).

Los depósitos de material zeolítico tienen lugar tanto en la superficie del soporte de alúmina, como en el contorno próximo del interior de las cavidades de los mismos. Al mismo tiempo en los polvos testigos y en el interior de los soportes se observan algunos aglomerados en forma de "rosas del desierto", (Fig. 5), cuya presencia se incrementa con el tiempo de tratamiento hidrotermal. En otros estudios

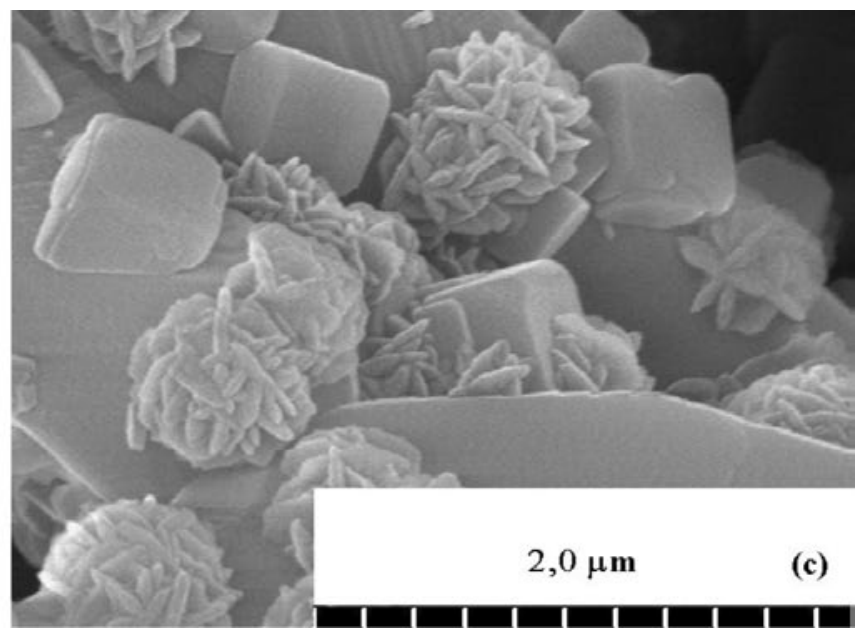

Fig.4. Imagen obtenida por MEB del soporte y de las zeolitas embebidas por síntesis hidrotermal "in situ” dentro de los soportes de alúmina: (a) vista de la sección transversal del soporte próximo a la superficie, (b) vistas de la sección transversal en un plano interior. 

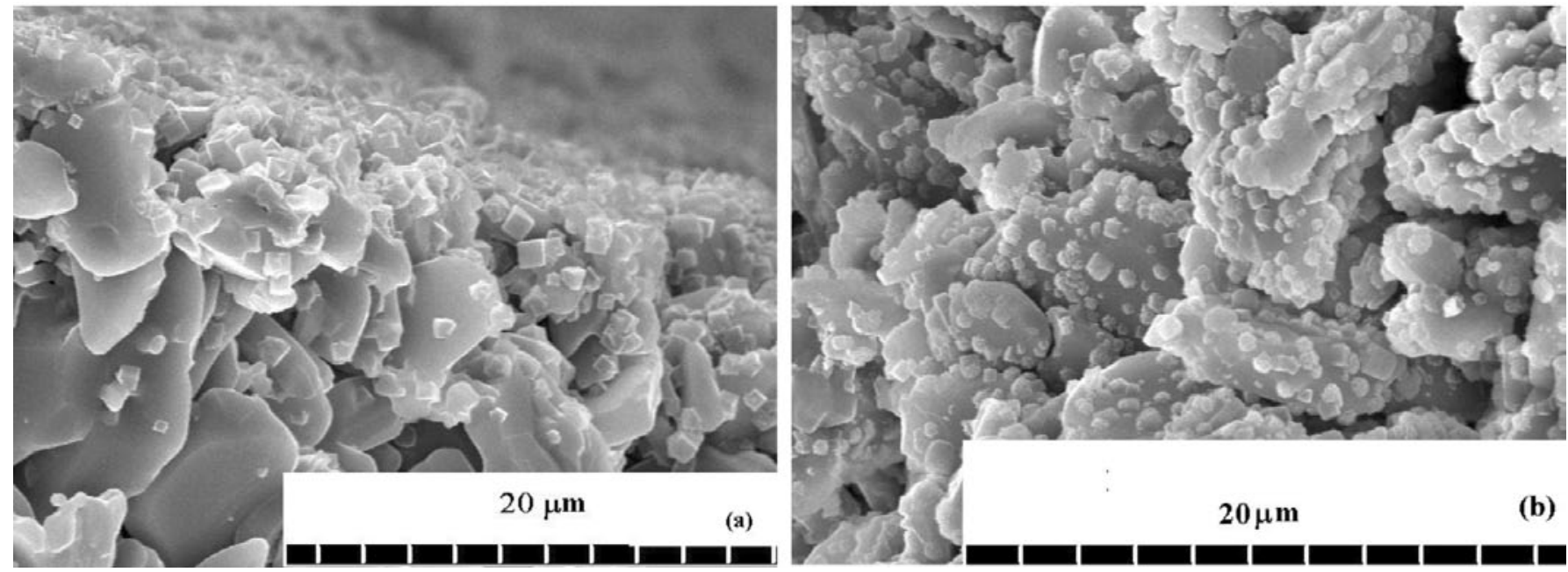

Fig. 5. Imagen por MEB de los polvos testigo obtenidos por síntesis hidrotermal "in situ" en presencia de los soportes de alúmina. Se observa el aspecto característico de cubos biselados y aglomerados en forma de "rosas del desierto"

realizados se evidencia que la formación de estos aglomerados es estimulada por la presencia misma de los soportes de alúmina. Por el momento se desconoce su naturaleza.

4.3 Eficiencia en la eliminación por intercambio iónico de iones $\mathrm{Cu}^{2+}$ en soluciones diluidas, en función del tiempo de contacto con la zeolita Na-LTA.

La Fig. 6 muestra los resultados de la eliminación por intercambio iónico de los iones de $\mathrm{Cu}^{2+}$ en soluciones diluidas, en función del tiempo de contacto con la zeolita Na-LTA.

Puede apreciarse que transcurridas 2 horas se han eliminado prácticamente todos los cationes $\mathrm{Cu}^{2+}$ de la solución, lo cual es comprensible si se tiene en cuenta la capacidad de intercambio teórica de la zeolita objeto de estudio.

La capacidad de intercambio iónico de una zeolita (CII) se reconoce como la magnitud característica del proceso de intercambio. Esta magnitud se valora en términos de cationes intercambiables equivalentes por gramo de zeolita, entendiendo como cationes intercambiables a aquellos que

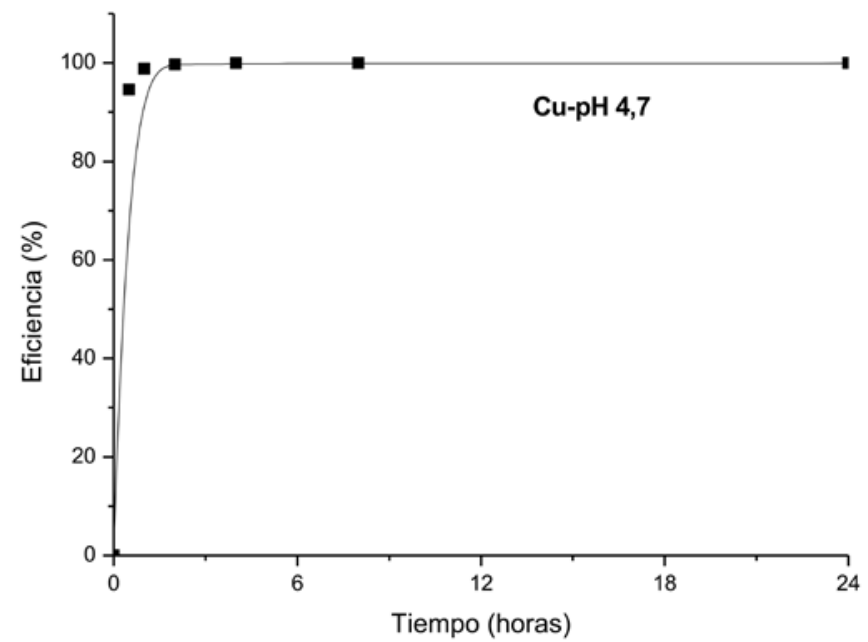

Fig. 6. Variación de la eficiencia de eliminación por intercambio iónico de iones $\mathrm{Cu}^{2+}$ en disoluciones diluidas, en función del tiempo de contacto con la zeolita Na-LTA, a una temperatura de $298 \pm 2 \mathrm{~K}$ y agitación a $180 \mathrm{rev} / \mathrm{min}$. con mayor o menor facilidad pueden ser reemplazados por otros cationes en soluciones acuosas sin afectar la estructura de la red.

En una zeolita la capacidad de intercambio iónico teórico depende de la concentración de cationes intercambiables y puede expresarse como:

$$
C I I=\frac{\text { Total cationes intercambiables }}{\text { Masa de la fórmula unidad }} \times 1000(\mathrm{meq} / \mathrm{g})
$$

En el caso de la zeolita $\mathrm{A}$, atendiendo a su fórmula química: $\left(\mathrm{Na}_{12}\left(\mathrm{Al}_{12} \mathrm{Si}_{12}\right) \mathrm{O}_{48} \cdot 27 \mathrm{H}_{2} \mathrm{O}\right.$

La CII teórica es $5.48 \mathrm{meq} / \mathrm{g}$ por tanto, la cantidad de iones $\mathrm{Cu}^{2+}$ presentes en la solución es muy inferior a la cantidad máxima que $1 \mathrm{~g}$ de zeolita $\mathrm{A}$ sería capaz de eliminar por intercambio $\left(0.174 \mathrm{~g}\right.$ de $\left.\mathrm{Cu}^{2+}\right)(26)$

La Fig. 7 muestra la comparación respecto del estudio por DRX de la fase activa zeolítica antes y después del intercambio iónico en la solución conteniendo iones de $\mathrm{Cu}^{2+}$.

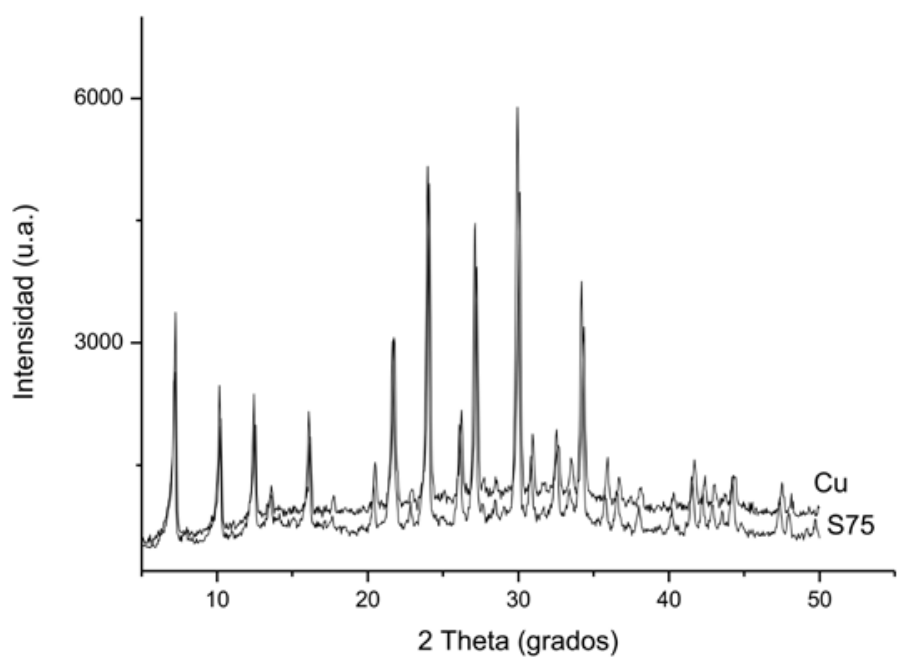

Fig. 7. Espectro de DRX de la fase activa antes y después de la interacción con los iones $\mathrm{Cu}^{2+}$ en la solución acuosa. 
En los difractogramas se observa la ausencia de cambios significativos en el ancho y en la intensidad de las reflexiones fundamentales, lo que está en correspondencia con la no manifestación de modificaciones en el tamaño de las partículas por efecto de la acción del pH del medio.

\section{CONCLUSIONES}

Se ha realizado el estudio preliminar del proceso de síntesis de zeolita A dentro de los soportes de alúmina y la valoración de la fase activa zeolítica para los procesos de eliminación de metales pesados.

Se observa que los cristales de zeolita son embebidos dentro de las cavidades de los soportes de alúmina, así como en la superficie del soporte por síntesis hidrotermal "in situ", sin el empleo de semillas.

La reacción para obtener zeolita Na-LTA se ha desarrollado satisfactoriamente a $378 \mathrm{~K}$, en un intervalo de $75 \mathrm{~min}$ con las siguientes relaciones molares:

$$
34 \mathrm{Na}_{2} \mathrm{O}: \mathrm{Al}_{2} \mathrm{O}_{3}: 3 \mathrm{SiO}_{2}: 462 \mathrm{H}_{2} \mathrm{O}
$$

La transición de zeolita tipo LTA a una SOD, más estable termodinámicamente, pero de menores aplicaciones potenciales, tanto como tamiz molecular como para intercambio iónico, tiene lugar a largos períodos de tratamiento hidrotermal.

A partir de los estudios de MEB se constata la no formación de una membrana real, pero si la presencia de cristales aglomerados y/o aislados en los poros de los cristales de alúmina próximos a la superficie. Estas aglomeraciones en el interior de los soportes se corresponden con los sitios que puede alcanzar el sol de la solución madre, durante el proceso de inmersión del soporte en la misma.

Se ha llevado a cabo la evaluación del material zeolítico desarrollado como fase activa para la eliminación de metales pesados con resultados satisfactorios, incluso en una solución de la sal con una concentración superior a la que se manifiesta en contaminaciones no agudas.

En las condiciones experimentales propuestas, mediante el empleo de una elevada relación de zeolitas/meq de iones en solución, valores superiores a 2 hacen rápido y efectivo el proceso de eliminación de metales pesados. El intervalo de 4 horas es suficiente para alcanzar el equilibrio del proceso de eliminación de estos metales, tal y como se demuestra en el presente trabajo.

\section{AGRADECIMIENTOS}

Este trabajo se ha realizado en el marco del proyecto 2009MA0008 del convenio bilateral CSIC-CNRST. Además ha sido financiado por la CICYT a través del proyecto MAT 201017753.

\section{BIBLIOGRAFÍA}

1. D. W. O'Connell; C. Birkinshaw; T.F. O'Dwyer. Heavy metal adsorbents prepared from the modification of cellulose: A review, Biores. Techn. 99, 6709-6724, 2008.

2. C.N. Mulligan, R. Yong, B.F. Gibbs, An evaluation of technologies for the heavy metal remediation of dredged sediments. Journal of Hazardous Materials 85, 145-163, 2001.

3. W. Franus, M. Franus, J. Latosińska, R. Wójcik, Bol. Soc. Esp. Cerám. Vidr., 50, 193-200, 2011

4. S.E. Bailey, T.J. Olin, R.M. Bricka, D.D. Adrian, A review of potentially lowcost sorbents for heavy metals. Wat. Res. 33, 11, 2469-2479, 1999.

5. S. Babel, T.A. Kurniawan, Low-cost adsorbent for heavy metals uptake from contaminated water: a review, J. Hazard. Mater. B97, 219-243, 2003.

6. A.G. García-Valles, S. Martínez, R. Terradas, J.M. Nogués, J. M., Heavy metal-rich wastes sequester in mineral phases through a glass-ceramic process. Chemosphere 68, 1946-1953. 2007.

7. A.. Addaou, A. Laajeb, A.Lahsini, J. Bentama, M. A. Rodriguez. Study of the combining adsorption-microfiltration process for the treatment of colored waters. Bol. Soc. Ceram. Vidr. 51, 165-168, 2012.

8. J. Kyziol-Komosinska, F. Barba, P. Callejas, C. Rosik-Dulewska. Beidellite and other natural low-cost sorbents to remove chromium and cadmium from water and wastewater.Bol. Soc. Esp. Ceram. Vidr. 49, 121-128, 2010.

9. N. Wook, K.Y., Hwang, K.Y., H. Bok Choi. Removal of Pb ion from water by magnetically modified zeolite, Minerals Engin. 19, 1452-1455, 2006.

10. R. Apiratikul, P. Pavasant, Sorption of $\mathrm{Cu}^{2+}, \mathrm{Cd}^{2+}$, and $\mathrm{Pb}^{2+}$ using modified zeolite from coal fly ash, Chem. Engin. J., 144, 245-258, 2008.

11. M. Pansini, C. Colella, D. Caputo, M. de' Gennaro, A. Langella, Evaluation of phillipsite as cation exchanger in lead removal from water, Micropor. Mater. 5, 357-364, 1996

12. Y. Sik Ok, J.E., Yang, Y. Seon Zhang, S. Jung Kimb, D. Young Chung, Heavy metal adsorption by a formulated zeolite-Portland cement mixture, J. of Hazard. Mater. 147, 91-96, 2007.

13. B. Bayati, A.A. Babalou, T. Karimi, Hydrothermal synthesis of nanostructure $\mathrm{NaA}$ zeolite: The effect of synthesis parameters on zeolite seed size and crystallinity, J. Eur. Ceram. Soc. 28, 2653-2657, 2008.

14. D.W. Breck, Zeolite Molecular Sieve, Structure, Chemistry and Use, E. Krieger Publishing Co, USA, 1994.

15. J. Peric, M. Trigo, N. Vukojevi!c Medvidovi, N., Removal of zinc, copper and lead by natural zeolite-a comparison of adsorption isotherms. Water Res. 38, 1893-1899, 2004.

16. M. Vaca Mier, R. López Callejas,R. Gehr, B.E. Jiménez Cisneros, P.J.J. Alvarez, Heavy metal removal with mexican clinoptilolite: Multi-component ionic exchang. Water Res. 35, 2, 373-378, 2001.

17. V.J. Inglezakis, H. Grigoropoulou, Effects of operating conditions on the removal of heavy metals by zeolite in fixed bed reactors, J. Hazard. Mater. B112 37-43, 2004.

18. H. Gold, C. Calmon, Treatment of industrial waste by ion exchange, AIChE Symposium. Series. 76, 60-67, 1980.

19. R. Cioffi R., L. Maffucci, G.Mirtone, L. Santoro, Feasibility of manufacturing building materials by recycling a waste from ion exchange process, Environ. Tech. 19, 1145-1150, 1998.

20. C. Cabrera, C. Gabaldón, P. Marzal, P., Sorption characteristics of heavy metal ions by a natural zeolite, J. Chem. Techn. \& Biotech., 80, 477-481, 2005.

21. J. S. Kim, M.A. Keane, The removal of iron and cobalt from aqueous solutions by ion exchange with Na-Y zeolite: batch, semi-batch and continuous operation, J. Chem. Techn. \& Biotech., 77, 633-640, 2002.

22. D. Escalante, D. Giraldo, M. Pinto, C. Pfaff, V. Sazo, M. Matjushin, B. Mendez, C.M. Lopez, F.J. Machado, J. Goldwasser, M.M. Ramirez de Agudelo, A study of the feasibility of incorporation of chromium into the molecular sieve framework: The transformation of 1-butene over Cr-silicoaluminophosphate molecular sieves. J. of Catalysis , 169, 176-187, 1997.

23. Ch. Baerlocher, Ch., and L.B. McCusker, Database of Zeolite Structures: http:/ / www.iza-structure.org/databases/.

24. E. Basaldella, P.G. Vázquez, F. Lucolano, D. Caputo, Chromium removal from water using LTA zeolites: Effect of pH. J. Coll. Interf. Sci., 313, 574-578, 2007.

25. S. Muhammad, E. Munawar, Nanocrystalline Zeolite Y: Synthesis and Heavy Metal Removal, J. Rekay. Kimia Lingkungan, 6, 55-62, 2007.

26. F. A. Mumpton,., (Chairman of the International Committee on Natural Zeolites) and GSA Resources Inc. (http://www.gsaresources.com/ cationexchange.htm).

27. M. R. Davis, Molecular Sieves Synthesis, Chemical Engineering California Institute of Technology. Pasadena, CA 91125, 2003.

28. M.J. Treacy, J.B. Higgins, Collection of Simulated XRD Powder Patterns for Zeolites, 4th. Edition, 2001. 